\title{
X Congreso Ibérico de Agroingeniería

\section{Proyecto LIFE Ammonia Trapping: planta piloto para la captura de amoniaco del purín}

\author{
M. C. García-González ${ }^{1}$, I. González García ${ }^{1}$, B. Riaño ${ }^{1}$, B. Molinuevo Salces ${ }^{1}$, D. Hernández \\ González ${ }^{1}$, M. Soto-Herranz' ${ }^{1}$, A. Correa-Guimarães ${ }^{2}$, L. M. Navas Gracia ${ }^{2}$, M. Sánchez- \\ Báscones $^{2}$ \\ 1 Instituto Tecnológico Agrario de Castilla y León. Ctra. Burgos, km. 119 47071, Valladolid, España. \\ 2 Departamento de Agricultura e Ingeniería Forestal, ETSIIAA, Universidad de Valladolid, Avenida de \\ Madrid 44, 34004 Palencia, España.
}

\begin{abstract}
Resumen: Las emisiones de amoniaco son uno de los contaminantes más importantes generados en las actividades agrícolas y ganaderas, estos sectores son los responsables de más del $93 \%$ de las emisiones de amoniaco en la UE. Dichas emisiones causan problemas medioambientales y de salud, por lo que la Unión Europea trata de reducirlas desde el año 2010 con la Directiva NEC 2001/81/CE sobre techos de emisiones, la cual ha sido revisada a través de la Directiva 2016/2284/EU. El proyecto Life Ammonia Trapping tiene como principal objetivo reducir las emisiones de amoniaco de granjas porcinas y avícolas, a través de la aplicación de una nueva tecnología de membranas permeables a los gases. En este trabajo se presentan resultados del primer estudio a escala piloto con la tecnología de membranas permeables a los gases, llevado a cabo en una granja porcina. De acuerdo con estos datos se ha conseguido una recuperación de amoníaco del $66,2 \%$ en el prototipo estudiado.
\end{abstract}

Palabras clave: amoniaco, emisiones, purines, prototipo, tecnología de membranas

\section{Introducción}

El sector ganadero, y especialmente el porcino, es un sector altamente competitivo, basado en sistemas de manejo de alta productividad y con alta demanda de recursos como materias primas para piensos, agua y energía. La especialización del sector en los últimos años se ha traducido en una mayor y mejor producción animal, con granjas de gran tamaño y menor número de granjas, por lo que España cuenta con la mayor cabaña ganadera de Europa, pero con menos granjas que hace unos años, en torno al 13\% [1]. Además, se suelen agrupar en zonas determinadas, lo cual tiene implicaciones ambientales graves debidas a determinados elementos contaminantes como los nitratos y el amoníaco.

En el caso de este último, la Unión Europea a través de su Directiva 2016/2284 (Directiva Techos o NEC Directive) [2] ha fijado límites máximos para las emisiones de gases a todos Estados miembros a partir de 2010 con el objetivo de mitigar la contaminación atmosférica. Para ello, se están desarrollando una serie de actuaciones, tales como la revisión del Protocolo de Gotemburgo de 2012 y la revisión de la Directiva de Techos, con una propuesta de techos de emisión en 2020 y 2030, así como niveles intermedios para 2025 que permitan hacer un seguimiento de las emisiones para conseguir el objetivo de 2030. En el caso del amoníaco, se han constatado cantidades de 378,7 y 375,3 Gg según los inventarios de 2005 y 2012 respectivamente, estableciéndose una previsión de 396,3 Gg en el año 2020 cuando el techo establecido en la UE es 


\section{CONGRESO IBÉRICO DE AGROINGENIERÍA \\ X CONGRESSO IBÉRICO DE AGROENGENHARIA \\ 3 - 6 septiembre 2019, Huesca - España}

de 365,0 Gg, tendencia que se agrava en el año 2030 en el cuál la previsión de emisión es de 393,7 $\mathrm{Gg}$ y el techo de 267,2 Gg [3]. El no cumplimiento de estos techos implica sanciones para España.

El sector agroganadero está directamente relacionado con la emisión de diferentes tipos de gases emitidos a la atmósfera, especialmente el $\mathrm{NH}_{3}$, que se genera por la descomposición de la urea y del ácido úrico contenidos en las excretas. $\mathrm{El} \mathrm{NH}_{3}$ puede formar aerosoles, acidificar suelos, contaminar con nitratos las aguas subterráneas y superficiales y causar eutrofización de esas aguas. Además, en la atmósfera contribuye a la formación de partículas PM 2.5. Todo ello justifica la adopción de medidas para su reducción, algunas de las cuales ya se están implementando, como son las relacionadas con la aplicación de las Mejores Técnicas Disponibles, las incluidas en el Código de Buenas Prácticas Agrarias o las relacionadas con la gestión del estiércol.

En este sentido, el proyecto Life Ammonia Trapping trata de contribuir a la reducción de las emisiones de amoniaco generadas por los residuos ganaderos que se producen en las granjas de ganado porcino y avícola, aplicando la tecnología novedosa de membranas permeables a los gases. A lo largo del proyecto se han diseñado y construido dos prototipos de captura de amoniaco, uno directamente del purín (prototipo de líquidos) y otro del aire que se encuentra en el interior de las naves de los animales (prototipo de gases).

El proceso de captura de $\mathrm{NH}_{3}$ se basa en la capacidad que tiene este compuesto para atravesar la membrana permeable a los gases pudiéndose recuperar en una solución ácida al otro lado de dicha membrana. Una vez el $\mathrm{NH}_{3}$ se concentra en la solución ácida, se combina con los $\mathrm{H}^{+}$para formar iones amonio $\left(\mathrm{NH}_{4}{ }^{+}\right)$no volátiles, convirtiéndose en una sal de amonio que se puede usar como fertilizante, y neutralizando el ácido, de manera que la solución producida no tendría que ser neutralizada para su uso posterior [4]. Este producto fertilizante se puede exportar fuera de la granja hacia regiones donde sea necesario, lo cual evitaría la contaminación nitrogenada al suelo, agua y al aire en zonas con exceso de nutrientes, como por ejemplo las zonas vulnerables.

El objetivo de este trabajo es presentar resultados del primer estudio a escala piloto con la tecnología de membranas permeables a los gases para la captura de amoniaco del purín. El estudio se ha llevado a cabo en una granja porcina dentro del marco del proyecto Life Ammonia Trapping, sin que haya terminado todavía la fase experimental del proyecto.

\section{Metodología}

\subsection{Descripción del prototipo.}

El prototipo está ubicado en una granja de ganado porcino de la provincia de Palencia (Figura 1). Consta de los siguientes elementos: 1) un tanque de 5,8 $\mathrm{m}^{3}$; 2) una bomba de alimentación del purín que introduce el purín desde la balsa de almacenamiento de la granja hasta el tanque de $5,8 \mathrm{~m}^{3}$; 3) 16 paneles de membrana que están sumergidos en el purín y dentro del tanque de $5,8 \mathrm{~m}^{3}$, cada panel contiene aproximadamente $50 \mathrm{~m}$ de membrana tubular; 4) una soplante; 5) una bomba de recirculación del ácido; 6) un tanque de 250 litros para el almacenamiento de la solución ácida; 7) una bomba de recirculación del purín; 8) dos sondas de $\mathrm{pH}$ y temperatura y 9) un sistema de control de los equipos (Figura 2). Este sistema de control regula el funcionamiento de la soplante de manera que cuando el $\mathrm{pH}$ del purín es inferior a 8,3 entra en funcionamiento la soplante para introducir aire y así subir el $\mathrm{pH}$ de nuevo. Esto es importante ya que el equilibrio $\mathrm{NH}_{3} / \mathrm{NH}_{4}{ }^{+}$está gobernado por el $\mathrm{pH}$ del purín y la temperatura. A mayor $\mathrm{pH}$ mayor $\mathrm{NH}_{3}$ en el purín, lo cual favorece su captura por la membrana. Asimismo, el $\mathrm{pH}$ del ácido se ha mantenido por debajo de 2 para favorecer también la captura de $\mathrm{NH}_{3}$. 


\section{CONGRESO IBÉRICO DE AGROINGENIERÍA \\ X CONGRESSO IBÉRICO DE AGROENGENHARIA \\ 3 - 6 septiembre 2019, Huesca - España}

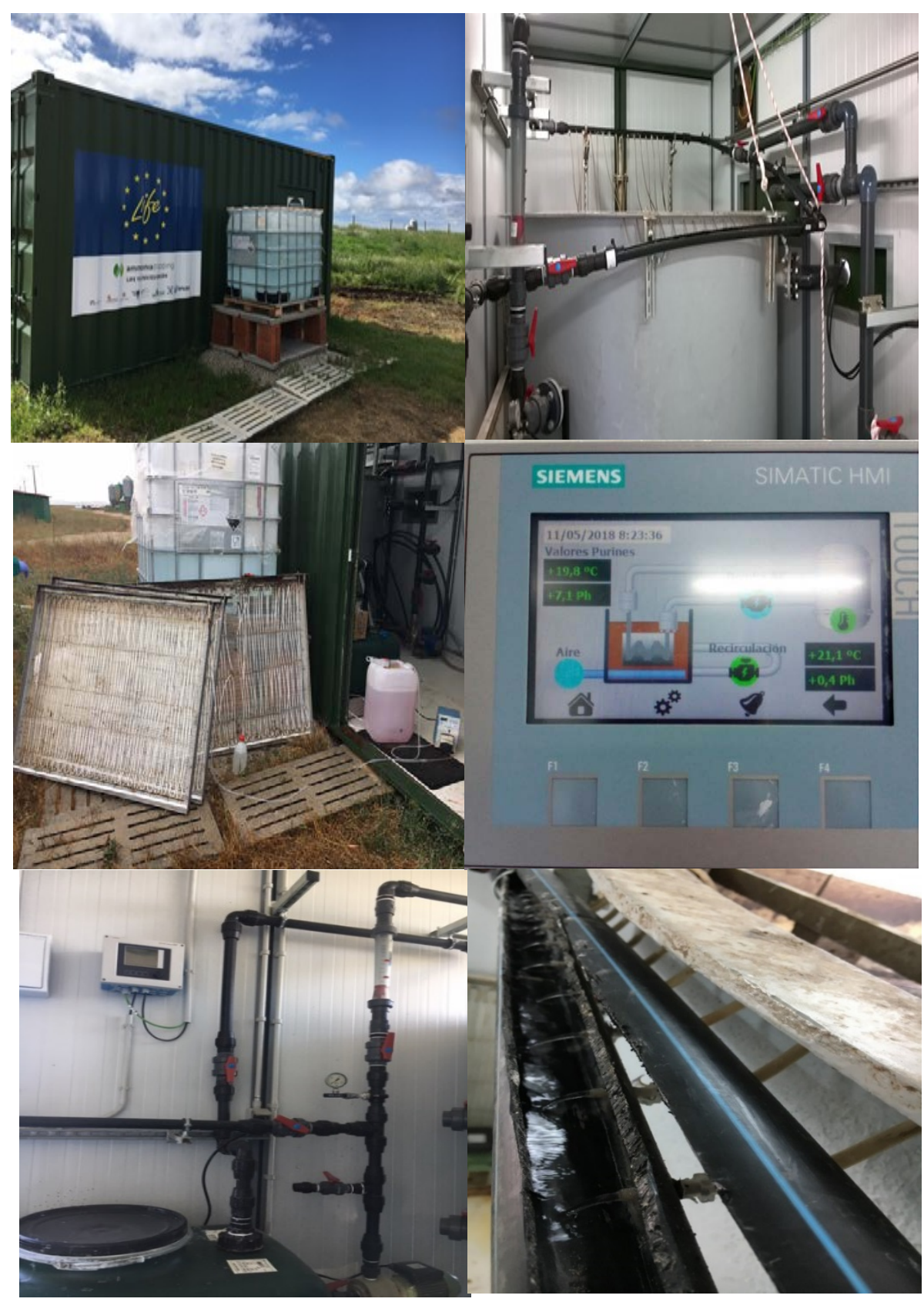

Figura 1. Vista general del prototipo, tanque de purín, paneles con las membranas, panel de control, tanque de solución ácida, salida de la solución ácida de cada panel.

\subsection{Muestreos y caracterización de las muestras.}

Los ensayos se han realizado por lotes, es decir, se introduce el purin en el tanque de $5,8 \mathrm{~m}^{3}$ y se mantiene el mismo purín durante 7-10 días. El volumen de purin tratado es de $5 \mathrm{~m}^{3}$ y el de solución ácida es de 150 L. Durante todo el periodo experimental se han recogido muestras diarias de purín y de solución ácida, se han transportado y mantenido refrigeradas a $4{ }^{\circ} \mathrm{C}$ hasta su análisis. Se han realizado los siguientes análisis: $\mathrm{pH}$, temperatura, TAN (nitrógeno amoniacal total) de los dos tipos de muestra. Al inicio y al final de cada lote se han hecho los siguientes análisis: alcalinidad total, sólidos totales (ST) y volátiles (SV), TAN, nitrógeno total (Nt), DQO total y soluble (demanda química de oxígeno). La alcalinidad total y el $\mathrm{pH}$ se han medido con un pHmetro Crison Basic 20 (Crison Instrumentos S.A., Barcelona). Los analisis de ST, SV, DQOt, DQOs, TAN y Nt se realizaron según la metodología del Standard Methods [5]. 


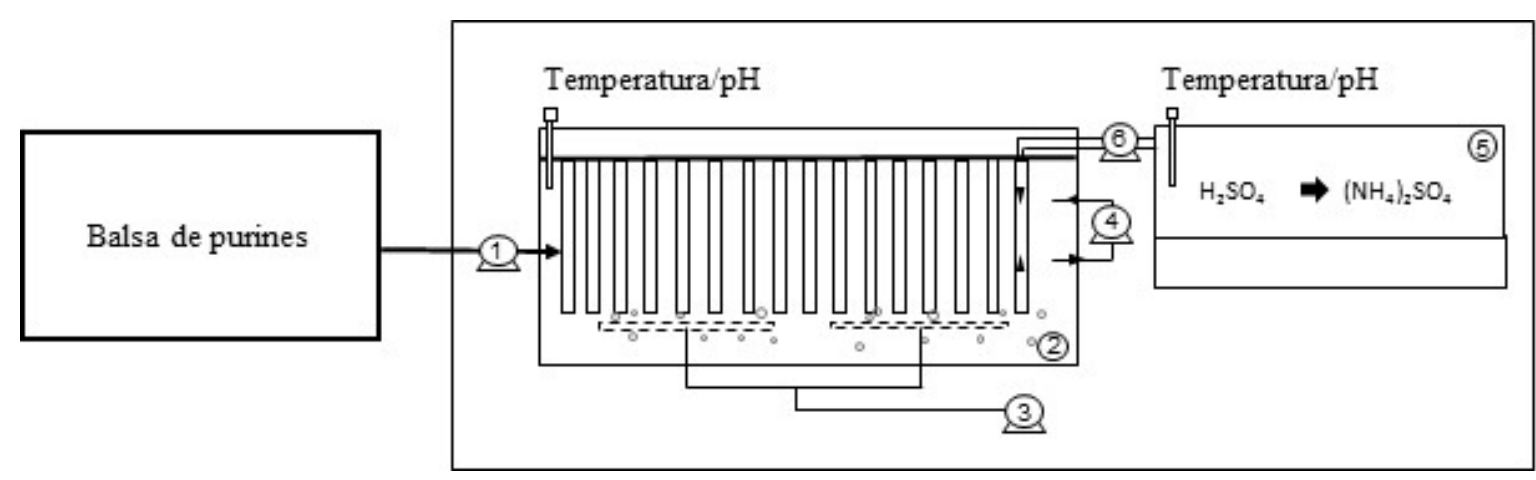

Figura 2. Esquema del prototipo de líquidos. 1) bomba de alimentación de purín, 2) aireadores, 3) soplante, 4) bomba de recirculación del purín, 5) tanque de solución ácida, 6) bomba de recirculación de la solución ácida.

\section{Resultados y discusión}

El prototipo está instalado en una granja de ganado porcino, el purín de la granja tiene un alto contenido en materia orgánica con $41,87 \mathrm{~g} \cdot \mathrm{L}^{-1}$ de DQO total y $30,42 \mathrm{~g} \cdot \mathrm{L}^{-1}$ de sólidos totales y $2,82 \mathrm{~g} \cdot \mathrm{L}^{-1}$ de nitrógeno amoniacal (Tabla 1), por lo que se considera un purín muy cargado.

Tabla 1. Caracterización química del purín al inicio y final del ensayo. Los datos son la media de seis muestreos, entre paréntesis se indican las desviaciones estándar.

\begin{tabular}{ccc}
\hline Parámetro & Inicial & Final \\
\hline $\mathrm{pH}$ & $7,26(0)$ & $8,49(0)$ \\
$\mathrm{DQOt}\left(\mathrm{g} \cdot \mathrm{L}^{-1}\right)$ & $41,87(3,24)$ & n.d \\
$\mathrm{ST}\left(\mathrm{g} \cdot \mathrm{L}^{-1}\right)$ & $30,42(2.23)$ & n.d \\
$\mathrm{TAN}(\mathrm{g} \cdot \mathrm{L}-1)$ & $2,82(0)$ & $1,92(0)$ \\
$\mathrm{Nt}\left(\mathrm{g} \cdot \mathrm{L}^{-1}\right)$ & $3,38(0,01)$ & n.d \\
\hline
\end{tabular}

n.d: no determinado

Según se observa en la figura 3, la concentración de TAN disminuyó en el purín desde 2,82 $\mathrm{g} \cdot \mathrm{L}^{-1}$ hasta $1,92 \mathrm{~g} \cdot \mathrm{L}^{-1}$ en un periodo de 7 días. Dicho TAN se recuperó en la solución ácida en una concentración de $18,6 \mathrm{~g} \cdot \mathrm{L}^{-1}$, lo que implica una concentración 6,6 veces superior al TAN del purín. En comparación con otros estudios llevados a cabo en nuestro laboratorio, en este ensayo se han obtenido mayores concentraciones de TAN en la solución ácida [4,6], debido a una mayor concentración de TAN en el tanque de purín. Hay que resaltar que la solución ácida ha sido la misma durante todo el ensayo, recirculándose constantemente dentro de las membranas. Esta solución está formada por ácido sulfúrico, que combinado con el $\mathrm{NH}_{3}$ del purín producen sulfato de amonio, que es un fertilizante comercial de uso general en agricultura. Por lo que el fin último de esta solución es su uso como fertilizante. Además, dado que la captura de TAN hace que el pH de la solución ácida aumente, cuando sea necesario reemplazar dicha solución no será necesaria su neutralización ya que ocurrirá debido al propio mecanismo de la reacción. 
X CONGRESO IBÉRICO DE AGROINGENIERÍA

X CONGRESSO IBÉRICO DE AGROENGENHARIA

3- 6 septiembre 2019, Huesca - España

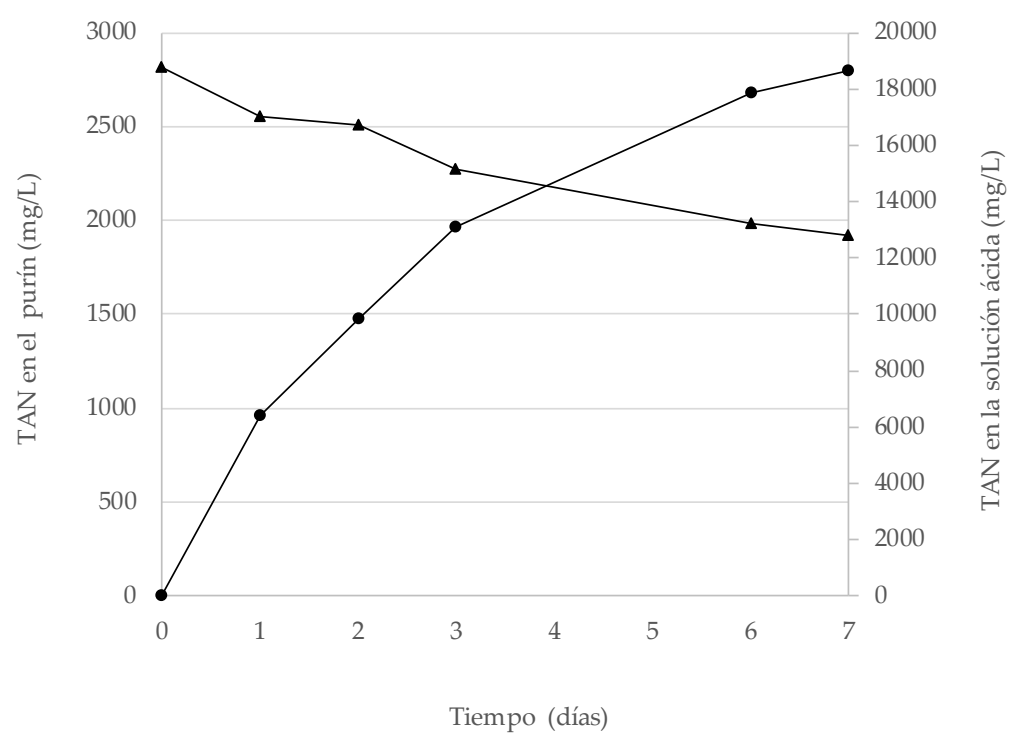

Figura 3. Eliminación de TAN del purín y recuperación de TAN en la solución ácida mediante la tecnología de membranas permeables a los gases.

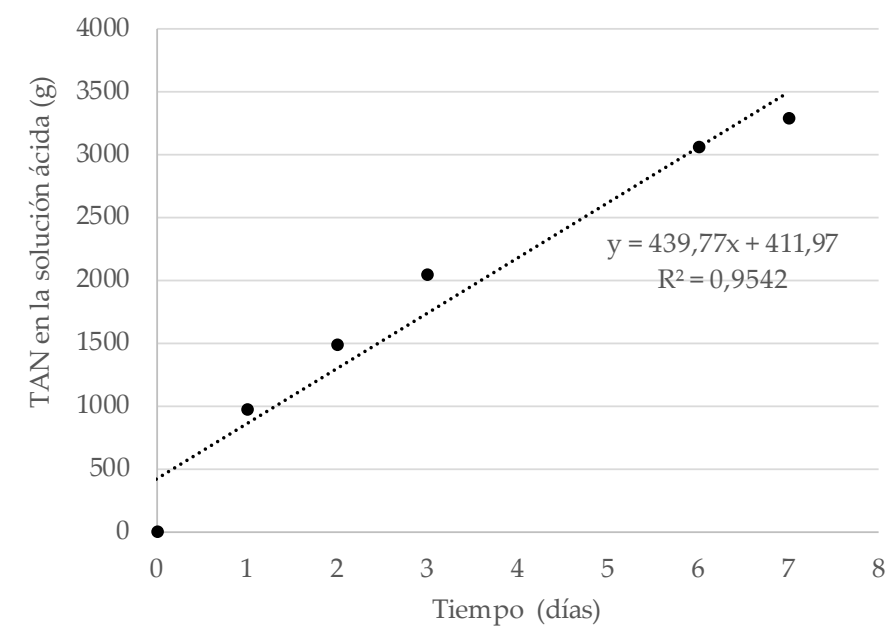

Figura 4. Masa de TAN recuperado en la solución ácida. Se representa la ecuación de primer orden y $\mathrm{R}^{2}$.

Como se muestra en la figura 4, la captura de TAN por la membrana ha sido lineal durante el periodo experimental, lo cual significa que la tasa de captura de TAN fue constante durante ese periodo, y que la concentración de TAN en el purín fue lo suficientemente alta para que la membrana lo capturase de forma continua. En estudios llevados a cabo en el laboratorio durante un periodo experimental mayor [4,6], la recuperación de TAN no ha sido lineal, si no que ha seguido una curva de segundo grado debido a la disminución del contenido de TAN en el tanque. En base a estos resultados, es importante mantener una concentración de TAN alta en el tanque para maximizar la captura de TAN por la membrana. 


\section{CONGRESO IBÉRICO DE AGROINGENIERÍA \\ X CONGRESSO IBÉRICO DE AGROENGENHARIA \\ 3 - 6 septiembre 2019, Huesca - España}

Tabla 2. Balance de masa de la recuperación de nitrógeno utilizando las membranas permeables

a los gases.

\begin{tabular}{cc}
\hline & Ensayo 1 \\
\hline TAN inicial en el purín $(\mathrm{g})$ & 14.001 \\
TAN final en el purín $(\mathrm{g})$ & 9.050 \\
TAN eliminado $(\mathrm{g})$ & 4.951 \\
TAN recuperado en la solución ácida $(\mathrm{g})$ & 3.279 \\
Porcentaje de TAN recuperado en el ácido $(\%)$ & 66,2 \\
Tasa de recuperación $(\mathrm{g}$ TAN·m-2·d-1) & 38,2 \\
\hline
\end{tabular}

A lo largo del ensayo realizado en el prototipo, se ha eliminado un del 45\% del TAN. Del TAN eliminado en el purín se ha recuperado el $66,2 \%$, lo cual implica un alto porcentaje en la reducción de las emisiones de amoniaco del purín (Tabla 2). En cuanto a la tasa de recuperación, en este ensayo se han alcanzado tasas de recuperación de $38,2 \mathrm{~g} \mathrm{TAN} \cdot \mathrm{m}^{-2} \cdot \mathrm{d}^{-1}$, que son algo mayores a las obtenidas en ensayos previos de laboratorio. Además, se han alcanzado valores de recuperación de $180 \mathrm{mg}$ TAN $\cdot \mathrm{m}^{-2} \cdot \mathrm{d}^{-1}$, que son mayores a la propuesta en uno de los objetivos del proyecto, que es capturar al menos $150 \mathrm{mg} \mathrm{TAN} \cdot \mathrm{m}^{-2} \cdot \mathrm{d}^{-1}$, por lo tanto, en este primer ensayo llevado a cabo con el prototipo, se ha logrado cumplir uno de los objetivos del proyecto.

\section{Conclusiones}

La recuperación de amoniaco del purín utilizando el prototipo diseñado y construido en el marco del proyecto Life Ammonia Trapping ha sido un éxito. Se han conseguido tasas de recuperación de $180 \mathrm{mg} \mathrm{TANL}^{-1} \mathrm{~d}^{-1}$, que son mayores a la propuesta en uno de los objetivos del proyecto. Además, se ha conseguido una recuperación de TAN del purín del $66,2 \%$. La solución de sulfato de amonio generada en el proceso es un producto fertilizante que será evaluado en el proyecto para su uso como fertilizante.

\section{Agradecimientos}

Este trabajo ha sido financiado con recursos procedentes de fondos FEDER a través del proyecto RTA201500060-C04-01, y del proyecto Life Ammonia Trapping Life15ENV/ES/284.

\section{Referencias}

1. Ministerio de Agricultura y Pesca, Alimentación y Medio Ambiente. 2018a. El sector de la carne de cerdo en cifras. Principales indicadores económicos 2017. Disponible en: https://www.mapa.gob.es/es/ganaderia/estadisticas/indicadoreseconomicoscarnedecerdo2017coment arios_tcm30-379728.pdf. Acceso 03/04/2019.

2. EC-European Commission (2016). Directive (EU) 2016/2284 of the European Parliament and of the Council of 14 December 2016 on the Reduction of National Emissions of Certain Atmospheric Pollutants, Amending Directive 2003/35/EC and Repealing Directive 2001/81/EC. OJL 344, 1-31.

3. Sanchez Bascones, M., García-González, M.C. Reducción de las emisiones de amoníaco y producción de fertilizantes de los residuos ganaderos. Retema, (2018), $\mathrm{n}^{\circ}$ de noviembre-diciembre, (22-27).

4. García-Gonzalez M. C., Vanotti M. B. Recovery of ammonia from swine manure using gas-permeable membranes: effect of waste strength and $\mathrm{pH}$. Waste Management (2015), 38, (455 - 461).

5. APHA. Standards Methods for the Examination of Water and Wastewater. 21st ed. Washington, D.C. American Public Health Association, American Water Works Association, Water Environment Federation (2005).

6. García-Gonzalez, M.C., Vanotti, M.B., Szogi, A.A. Recovery of ammonia from swine manure using gaspermeable membranes: Effect of aeration. Journal of Environmental Management (2015), 152 (19-26). 